\title{
Models for Autonomously Motivated Exploration in Reinforcement Learning*
}

\author{
Peter Auer ${ }^{1}$, Shiau Hong Lim $^{1}$, and Chris Watkins ${ }^{2}$ \\ 1 Chair for Information Technology, Montanuniversität Leoben, Austria \\ http://institute.unileoben.ac.at/infotech \\ 2 Department of Computer Science, Royal Holloway University of London, UK \\ http://www.rhul.ac.uk/computerscience
}

\begin{abstract}
One of the striking differences between current reinforcement learning algorithms and early human learning is that animals and infants appear to explore their environments with autonomous purpose, in a manner appropriate to their current level of skills. An important intuition for autonomously motivated exploration was proposed by Schmidhuber [12]: an agent should be interested in making observations that reduce its uncertainty about future observations.

However, there is not yet a theoretical analysis of the usefulness of autonomous exploration in respect to the overall performance of a learning agent. We discuss models for a learning agent's autonomous exploration and present some recent results. In particular, we investigate the exploration time for navigating effectively in a Markov Decsion Process (MDP) without rewards, and we consider extensions to MDPs with infinite state spaces.
\end{abstract}

Keywords: Reinforcement learning, autonomous exploration, intrinsic rewards.

\section{References}

1. Schmidhuber, J.: A Possibility for Implementing Curiosity and Boredom in ModelBuilding Neural Controllers. In: Meyer, J.A., Wilson, S.W. (eds.) International Conference on Simulation of Adaptive Behavior: From Animals to Animats, pp. 222-227. MIT Press, Cambridge (1991)

2. Schmidhuber, J.: Developmental Robotics, Optimal Artificial Curiosity, Creativity, Music, and the Fine Arts. Connection Science 18(2), 173-187 (2006)

\footnotetext{
* The full version of this paper is published in the Proceedings of the 22nd International Conference on Algorithmic Learning Theory, Lecture Notes in Artificial Intelligence Vol. 6925.
} 